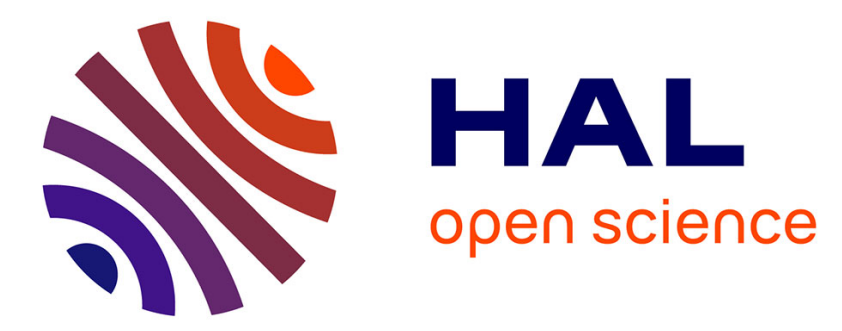

\title{
Prévalence des symptômes musculo-squelettiques multi-sites dans une population de travailleurs salariés
}

Elsa Parot-Schinkel, Alexis Descatha, Catherine Ha, Audrey Petit, Annette Leclerc, Yves Roquelaure

\section{- To cite this version:}

Elsa Parot-Schinkel, Alexis Descatha, Catherine Ha, Audrey Petit, Annette Leclerc, et al.. Prévalence des symptômes musculo-squelettiques multi-sites dans une population de travailleurs salariés. 14ème colloque de l'Aderest, Mar 2012, La Rochelle, France. 73 (5), pp.726-7, 2012, Archives des maladies professionnelles et de l'environnement. hal-02929295

\section{HAL Id: hal-02929295 \\ https://hal.science/hal-02929295}

Submitted on 3 Sep 2020

HAL is a multi-disciplinary open access archive for the deposit and dissemination of scientific research documents, whether they are published or not. The documents may come from teaching and research institutions in France or abroad, or from public or private research centers.
L'archive ouverte pluridisciplinaire HAL, est destinée au dépôt et à la diffusion de documents scientifiques de niveau recherche, publiés ou non, émanant des établissements d'enseignement et de recherche français ou étrangers, des laboratoires publics ou privés. 
1 UNAM ; Université d'Angers ; CHU Angers, IFR132, UPRES 4336, LEEST, Angers 2 Inserm U1018, Villejuif

${ }^{3}$ Département Santé Travail, Institut de veille sanitaire, Saint-Maurice

\section{Objectif}

Les troubles musculo-squelettiques (TMS) d'origine professionnelle représentent l'une des questions les plus préoccupantes en santé au travail. Bien que la très grande majorité des données de prévalence disponibles portent sur les TMS définis par localisation anatomique, un nombre croissant de travaux témoignent de la faible prévalence des symptômes limités à un site anatomique spécifique.

L'objectif de ce travail est de décrire la prévalence et les caractéristiques des symptômes musculo-squelettiques (SMS) multi-sites dans un large échantillon de travailleurs salariés français.

\section{Méthode}

Cette étude a été réalisée à partir des données de surveillance de l'enquête transversale (2002-2005) du réseau pilote de surveillance épidémiologique des TMS mis en place par l'Institut de veille sanitaire dans les Pays de la Loire ${ }^{1}$.

\section{Population}

- Travailleurs salariés dans la région des Pays de la Loire

- Inclus par tirage au sort par 83 médecins du travail volontaires au cours de visites périodiques

\section{Données}

- Les données relatives aux SMS ont été renseignées au moyen de l'auto-questionnaire standardisé dit « Nordique ».

- Deux définitions des SMS ont été utilisées dans ce travail :

- SMS déclarés au cours de 12 derniers mois

- SMS pendant au moins 30 jours (subaigus) déclarés au cours de 12 derniers mois

- Dans les analyses par région anatomique (rachis, membre supérieur et membre inférieur), la nuque est considérée comme une atteinte du membre supérieur.

- Les SMS multi-sites sont définies par la présence de SMS déclarés sur au moins deux sites anatomiques.

\section{Analyse statistique}

- Les analyses ont été réalisées séparément chez les hommes et les femmes pour étudier les éventuelles différences liées au genre.

- Les prévalences ont été calculées en divisant le nombre de sujets déclarant des symptômes (unilatéraux ou bilatéraux) pour le site d'intérêt sur le nombre total de sujets répondants, leurs intervalles de confiance à $95 \%$ ont également été calculés.

- Les comparaisons des données catégorielles ont été réalisées au moyen du test du chi-deux.

\section{Résultats}

Un peu moins de $10 \%$ des travailleurs vus dans lors des consultations sélectionnées au cours de la procédure d'échantillonnage à 2 niveaux (environ $2 \%$ des consultations réalisées par les médecins du travail du réseau) n'ont pas été inclus (absents, refus ou déjà inclus).

\section{Échantillon étudié}

Entre 2002 et 2005, 3710 travailleurs salariés (58 \% hommes, âge moyen $=38.7 \pm 10.3$ ans) ont été inclus.

La représentativité de l'échantillon étudié par rapport à la population active de la région était satisfaisante ${ }^{2}$

\section{Prévalences des SMS (Tableau)}

La prévalence des SMS au cours des 12 derniers mois était de $83,8 \%$ avec un intervalle de confiance à $95 \%$ de $[82,8-85,3]$ pour les hommes et de $83,9 \%[82,0-85,7]$ pour les femmes.

La prévalence des SMS d'au moins 30 jours au cours des 12 derniers mois était de $32,8 \%[30,9-34,8]$ pour les hommes et de $37,3 \%[34,9-$ $39,7]$ pour les femmes $(p=0,005)$.

\section{Prévalences des SMS multi-sites (Tableau)}

- 2/3 des salariés déclaraient des SMS multi-sites.

- 1/5 des salariés déclaraient des SMS d'au moins 30 jours multi-sites.
Tableau : Prévalences (\%) et intervalles de confiance à 95\% (IC) des symptômes musculo-squelettiques (SMS) au cours des 12 derniers mois

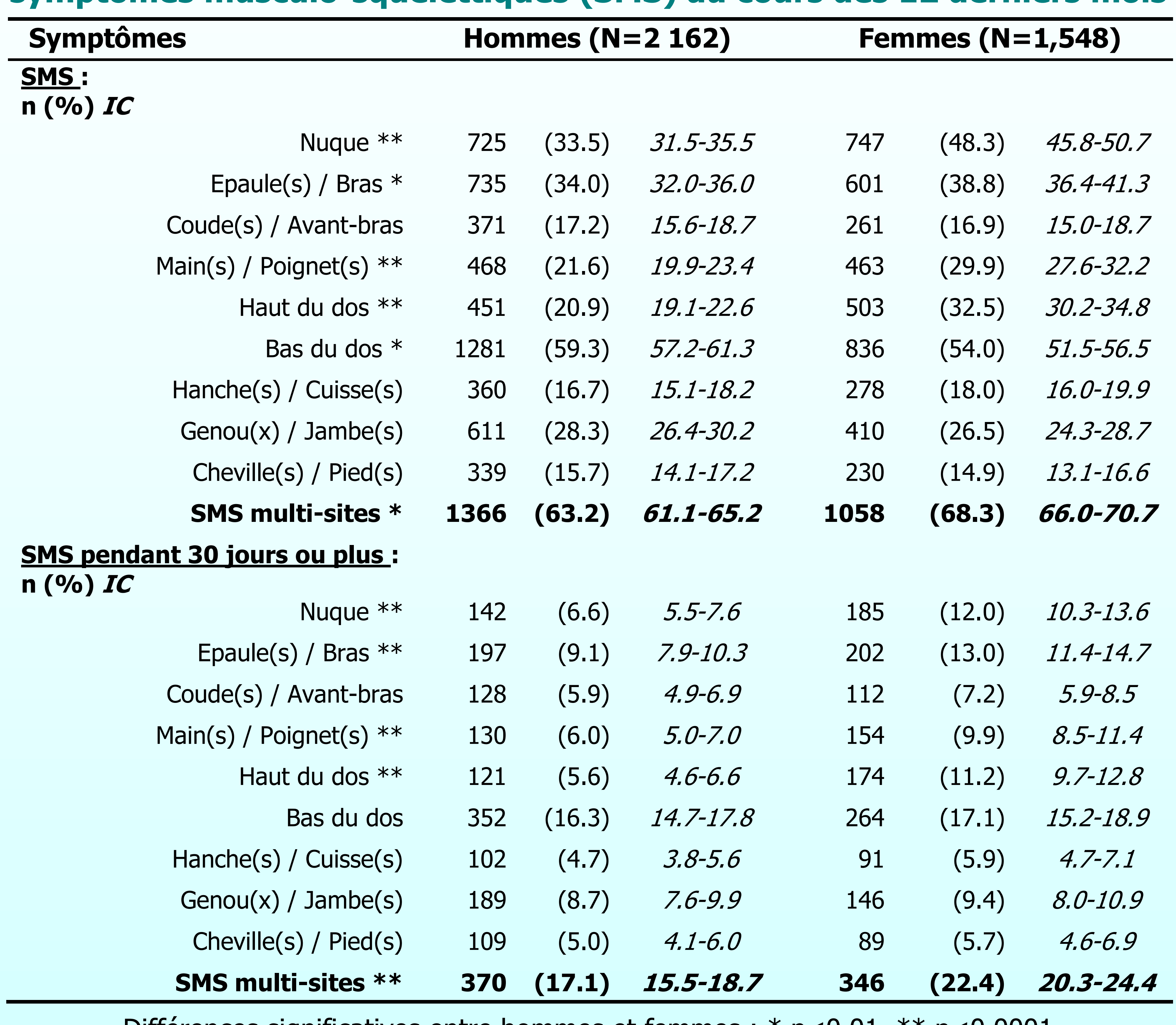

Différences significatives entre hommes et femmes : $* p<0.01, * * p<0.0001$

- $30,1 \%$ des salariés déclaraient des SMS sur $\geq 4$ sites anatomiques $(27,3 \%$ des hommes et $34,0 \%$ des femmes**), $10 \%$ déclarait des SMS sur $\geq 6$ sites $(8,2 \%$ des hommes et $12,7 \%$ des femmes**).

- Les salariés étaient $6,3 \%$ à rapporter des SMS d'au moins 30 jours sur $\geq 4$ sites anatomiques ( $4,9 \%$ des hommes et $8,3 \%$ des femmes*), les femmes étaient $4,5 \%$ à rapporter des SMS d'au moins 30 jours sur $\geq 5$ sites $(2,0 \%$ pour les hommes**).

- Les sites anatomiques les plus fréquemment associés à d'autres SMS étaient le haut du dos, la hanche, le coude et la nuque.

\section{SMS par région anatomique}

- Un tiers des sujets (31,3\% des hommes et 34,2\% des femmes) déclaraient la présence de SMS sur deux régions anatomiques au cours des 12 derniers mois (symptômes conjoints du rachis et du membre supérieur pour $2 / 3$ d'entre eux) et $27,2 \%$ déclaraient des symptômes au niveau des trois régions anatomiques.

- Plus de $90 \%$ des SMS multi-sites concernaient 2 régions anatomiques ou plus ( $91,9 \%$ pour les hommes et $90,6 \%$ pour les femmes).

- $9 \%$ des hommes et $12 \%$ des femmes* déclaraient la présence de SMS sur deux régions anatomiques pendant au moins 30 jours avec une prédominance des atteintes conjointes du rachis et du membre supérieur ( $51,3 \%$ des atteintes localisées à deux régions).

- Près de $80 \%$ des SMS multi-sites d'au moins 30 jours concernaient 2 régions anatomiques ou plus $(77,0 \%$ pour les hommes et $77,7 \%$ pour les femmes).

\section{Conclusion}

Cette étude confirme l'importance des atteintes multi-sites, y compris pour les atteintes d'au moins 30 jours. Ces données témoignent également d'une plus grande étendue des SMS chez les femmes. Compte tenu du mauvais pronostic associé à ces SMS multi-sites, il est essentiel de poursuivre les travaux sur cette thématique afin de mieux identifier les différents profils d'atteintes multi-sites et leurs déterminants.

\section{References}

${ }^{1}$ Ha C, Roquelaure $Y$, Leclerc A, Touranchet A, Goldberg M, Imbernon E. The French Musculoskeletal Disorders Surveillance Program: Pays de la Loire Network. Occup Environ Med 2009;66:471-9.

2 Roquelaure $Y$, Ha C, Leclerc A, Touranchet $A$, Sauteron M, Melchior M, Imbernon $E$ Goldberg M: Epidemiologic surveillance of upper-extremity musculoskeletal disorders in the working population. Arthritis Rheum 2006, 55:765-778.

Contact: Elsa Parot (ElParot@chu-angers.fr)

LEEST - UA InVS - IFR 132 - UPRES EA 4336 Faculté de Médecine, Rue Haute de Reculée 49045 ANGERS Cedex 01 